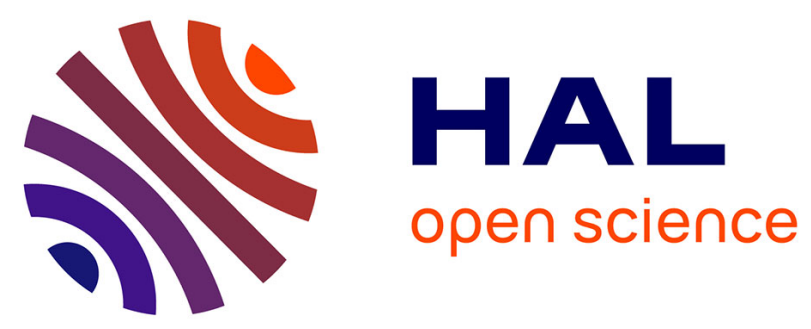

\title{
Dynamiques migratoires et genre : une approche de l’immigration féminine espagnole en Algérie (1939-1962)
}

Ludivine Thouverez

\section{To cite this version:}

Ludivine Thouverez. Dynamiques migratoires et genre : une approche de l'immigration féminine espagnole en Algérie (1939-1962). Mémoire(s), identité(s), marginalité(s) dans le monde occidental contemporain. Cahiers du MIMMOC, 2020, Circulations migratoires féminines dans l'espace hispanique et lusophone contemporain: vers une émancipation, 21-2020, 10.4000/mimmoc.4576 . hal-03237588

\section{HAL Id: hal-03237588 \\ https://hal.science/hal-03237588}

Submitted on 9 Jul 2021

HAL is a multi-disciplinary open access archive for the deposit and dissemination of scientific research documents, whether they are published or not. The documents may come from teaching and research institutions in France or abroad, or from public or private research centers.
L'archive ouverte pluridisciplinaire HAL, est destinée au dépôt et à la diffusion de documents scientifiques de niveau recherche, publiés ou non, émanant des établissements d'enseignement et de recherche français ou étrangers, des laboratoires publics ou privés. 
Mémoire(s), identité(s), marginalité(s) dans

Cahiers du MIMMOC

$21 \mid 2020$

Circulations migratoires féminines dans l'espace hispanique et lusophone contemporain: vers une émancipation

Dynamiques migratoires et genre : une approche de l'immigration féminine espagnole en Algérie (1939-1962)

\section{Ludivine THOUVEREZ}

\section{(2) OpenEdition Journals}

Electronic version

URL: https://journals.openedition.org/mimmoc/4576

DOI: $10.4000 /$ mimmoc.4576

ISSN: 1951-6789

Publisher

Université de Poitiers

Brought to you by Université de Poitiers

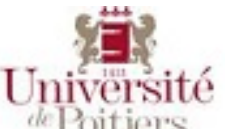

Electronic reference

Ludivine THOUVEREZ, "Dynamiques migratoires et genre : une approche de l'immigration féminine espagnole en Algérie (1939-1962)", Mémoire(s), identité(s), marginalité(s) dans le monde occidental contemporain [Online], 21 | 2020, Online since 15 August 2020, connection on 21 June 2021. URL: http://journals.openedition.org/mimmoc/4576 ; DOI: https://doi.org/10.4000/mimmoc.4576

This text was automatically generated on 21 June 2021.

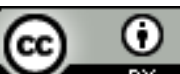

Mémoire(s), identité(s), marginalité(s) dans le monde occidental contemporain - Cahiers du MIMMOC est mis à disposition selon les termes de la licence Creative Commons Attribution 4.0 International. 


\title{
Dynamiques migratoires et genre : une approche de l'immigration féminine espagnole en Algérie (1939-1962)
}

\author{
Ludivine THOUVEREZ
}

\section{Introduction}

1 Jusqu'à la fin des années 1980, la question du genre fut relativement absente de la production scientifique consacrée aux dynamiques migratoires contemporaines. Longtemps considérées comme de simples «suiveuses ", " contraintes » d'abandonner leur pays en raison de la misère, les migrantes furent ainsi frappées d'un double phénomène d'invisibilité sociale: par les autorités publiques, d'une part; et par une communauté scientifique influencée par une vision androcentriste du réel, d'autre part. Ce constat est d'autant plus paradoxal que les femmes constituent depuis le milieu $\mathrm{du} \mathrm{XX} \mathrm{X}^{\mathrm{e}}$ siècle, près de la moitié des flux migratoires internationaux.

2 Si les départs des Espagnols vers les Amériques sont bien connus et documentés, ceux en direction du Maghreb font l'objet de peu d'informations. Tout au long des 132 ans de présence française en Algérie (1830-1962), plusieurs dizaines de milliers de personnes issues de la péninsule ibérique décidèrent pourtant de s'y établir, contribuant ainsi à forger l'identité d'un peuple qu'Albert Camus qualifiait d' « union des différences ${ }^{1}$ ».

Cet article entend présenter les caractéristiques de l'immigration féminine espagnole en Algérie entre 1939 et 1962. À partir de sources issues des Archives Générales de l'Administration espagnole (AGA) de la ville d'Alcalá de Henares, nous tenterons d'identifier les facteurs ayant entraîné le départ, l'établissement géographique et l'intégration en Algérie; et de déterminer si la migration de ces femmes vers une société coloniale suppose une redéfinition du statut social qui leur était assigné dans leur pays d'origine. 


\section{Femmes et archives : une infime présence}

Les conditions d'existence des Européens établis en Algérie coloniale ont commencé à susciter l'intérêt des historiens au début des années 1980. Christian Florès et JeanJacques Jordi furent, en France, les premiers à s'intéresser à la contribution des Espagnols à cette société, qui comptait un million d'Européens pour huit millions de Musulmans à la veille de l'indépendance ${ }^{2}$. Les travaux de Gérard Crespo et Anne Dulphy vinrent compléter leurs recherches ${ }^{3}$. En Espagne, l'historien Juan Bautista Vilar est l'auteur d'une importante production sur cette question ${ }^{4}$. Ses travaux influencèrent ceux de jeunes chercheurs alicantins ou valenciens (descendants pour la plupart de familles de migrants) qui, dans les années 2000 , envisagèrent le phénomène à travers un prisme local ou régional ${ }^{5}$.

5 Il convient de souligner qu'aucune de ces études n'envisage la dimension sexuée de l'émigration vers l'Algérie. Et cela, malgré l'émergence de travaux relatifs au genre dans les dynamiques migratoires depuis le milieu des années $80^{6}$. La thèse de Claudine Robert-Guiard, soutenue en 2005, contribue néanmoins à combler ce vide et à sortir les Européennes d'Algérie de l'invisibilité sociale ${ }^{7}$. L'historienne soulève également une question fondamentale: «ces Européennes [Françaises, Espagnoles, Italiennes, Allemandes ou Maltaises] étaient à la fois femmes et colonisatrices. Or coloniser, c'est conquérir, imposer, soumettre. C'est détenir une autorité considérée comme éminemment masculine ». L'historienne s'interroge donc sur la place des femmes dans l'articulation des rapports coloniaux de 1830 à la Seconde Guerre mondiale.

6 Cette étude s'inscrit dans la continuité des travaux de C. Robert-Guiard et vise, notamment, à comparer les données recueillies dans les archives françaises par les auteurs mentionnés ci-dessus avec celles, disponibles, aux Archives Générales de l'Administration (AGA) d'Alcalá de Henares. Dans la mesure où la période retenue pour cette étude est marquée par la Guerre civile espagnole, la Seconde Guerre mondiale et la guerre d'Algérie, une attention particulière sera portée aux documents de la Surreté de l'État et du Ministère des Affaires étrangères.

7 Des récits mémoriels complètent également ces sources primaires car les informations de l'administration espagnole sont souvent lacunaires. Contrairement aux autorités françaises, qui établissent un recensement régulier de la population résidente en Algérie, le Ministère des Affaires étrangères espagnol n'établit pas de registre de ses ressortissants à l'étranger. Les informations de l'AGA sont donc regroupées par thème (correspondance entre consulats, intérêts économiques, réfugiés politiques, etc.) et se présentent de manière éparpillée, de sorte qu'un recoupement systématique est nécessaire. De nombreux documents sont absents: ils ont été soit conservés dans d'autres archives, soit perdus ou détruits lors de l'indépendance de l'Algérie ou de la Transition, soit tout simplement non encore recensés. La plupart concernent le consulat d'Oran, et très occasionnellement ceux d'Alger et de Sidi-Bel-Abbes. En revanche, aucun document ne provient du consulat de Constantine.

8 À ces difficultés s'ajoutent celles liées au genre même de l'objet de l'étude :

Qu'il existe un problème de sources inhérent à l'histoire des femmes est maintenant bien connu. Les différents ouvrages écrits sur les femmes depuis les années 70 insistent sur le 'silence des archives'. Les archives publiques ne retiennent, comme 
le nom l'indique, que les documents publics relatifs surtout à la politique et à

l'économie, domaines dont les femmes ont été pendant longtemps exclues ${ }^{8}$.

La puissance maritale, établie par le Code civil espagnol de 1889 et renforcée par celui de 1938 (alors qu'elle est supprimée en France), a aussi pour conséquence que la femme vit sous la tutelle de son mari jusqu'au début des années 1970. Dans ces circonstances, rares sont les passeports ou documents établis au nom d'une femme, ce qui complique davantage le travail de l'historien. Néanmoins, le genre féminin est visible dans les registres d'état civil, ainsi que dans certaines correspondances dont le contenu sera évoqué plus loin.

\section{Un siècle de migrations vers l'Algérie}

10 Le cycle migratoire vers l'Algérie commence dès les premières années de la conquête militaire française en 1830. Malgré l'insécurité et les épidémies, de nombreux Espagnols quittent la péninsule pour rejoindre la colonie, qui présente l'avantage de n'être qu'à quelques heures de balancelle des côtes levantines. Contrairement aux Amériques, l'Afrique du Nord permet un départ à moindre coût et un retour facile en cas d'échec. Les raisons qui poussent les Espagnols à émigrer sont multiples. Pauvreté endémique, démographie excédentaire, prolétariat agricole, guerres carlistes ou encore troubles républicains de 1873, conduisent à la première grande vague d'émigration espagnole en Algérie. Celle-ci atteint son apogée entre 1882 et 1886, avec un nombre moyen de 15000 départs par an.

11 Embarquant dans les ports d'Alicante et d'Almería, les péninsulaires voyagent en groupe, s'établissent dans les plaines ou le long des côtes algériennes, et constituent des îlots de regroupement qui facilitent l'entraide et la mise en place de chaînes migratoires. Affectés au défrichage des terres et à des emplois subalternes (gardien de troupeau, charbonnier, mineur, cantinière, lavandière, épicière, etc.), hommes et femmes de la région de Valence et des îles Baléares sont appréciés pour leur « sobriété » et leur " endurance ${ }^{9}$ ». L'armée française s'inquiète toutefois du fait que la colonisation de peuplement soit laissée aux " gueux d'Espagne, d'Italie et de Malte ${ }^{10}$ " plutôt qu'à des familles de militaires français. Il faut dire qu'à cette époque, le nombre d'étrangers atteint presque celui des Français et qu'il le dépasse même lors du recensement de 1881 (tableau 1).

\begin{tabular}{|l|l|l|l|l|}
\hline Année & Français & Espagnols & Autres nationalités & Total Européens \\
\hline $\mathbf{1 8 7 6}$ & 189677 & 92510 & 62562 & 344749 \\
\hline $\mathbf{1 8 8 1}$ & 195418 & 114320 & 102697 & 412435 \\
\hline $\mathbf{1 8 9 6}$ & 366900 & 157560 & 54020 & 578480 \\
\hline
\end{tabular}

Tableau 1. Présence des Européens en Algérie. Source : Annuaires statistiques du gouvernement français.

12 Profitant de l'échec des colons français sur le sol africain, les Espagnols rachètent les terres abandonnées et les valorisent grâce au savoir-faire acquis dans leur pays. Des dizaines d'exploitations agricoles passent alors aux mains de la communauté, tel que 
l'explique la romancière Andrée Montero, petite-fille de viticulteurs alicantins, dans le roman autobiographique Río Salado ${ }^{11}$. En milieu urbain, en revanche, les emplois occupés par les Espagnols demeurent peu qualifiés et leur permettent tout au mieux de (sur)vivre, et très exceptionnellement de s'enrichir.

L'émigration espagnole présente plusieurs particularités. La première réside dans le fait qu'à la fin du XIX siècle, les femmes sont plus nombreuses que les hommes ${ }^{12}$. La deuxième est que le caractère familial de la migration semble avoir moins pesé dans la décision de partir que pour les autres communautés. L'analyse des mortuaires de la période 1846-1858 permet à C. Robert-Guiard d'affirmer que les Espagnoles partent jeunes et célibataires ou bien "âgées ", c'est-à-dire, une fois leurs enfants élevés au pays.

14 L'historienne observe que $57 \%$ sont célibataires lors de leur décès et que leur taux d'activité est important : il atteint 50\% chez les célibataires et 39\% chez les veuves. En l'absence d'autres informations, on peut supposer qu'une grande partie des immigrées se consacrent aux métiers du care (domestique, cuisinière, nourrice, lavandière, etc., métiers impliquant de loger chez ses patrons) et qu'une autre partie travaillent dans le commerce et l'industrie, comme dans les usines March et Bastos qui emploient notamment des cigarières espagnoles. Une dernière partie se consacre enfin à la prostitution. Selon un rapport du fonctionnaire Édouard-Adolphe Duchesne, les Espagnoles représentent $75 \%$ et $25 \%$ des «filles publiques » d'Oran et d'Alger durant les vingt premières années de la colonisation. Le fonctionnaire note également qu'un grand nombre de femmes (non recensées par les autorités) s'y consacrent de manière ponctuelle et clandestine, afin d'échapper à la misère ou d'améliorer leurs conditions de vie ${ }^{13}$.

15 Le récit mémoriel Las Golondrinas ou les trois scuurs d'Almería, de l'écrivain Denis Nuñez, apporte de nombreuses informations sur cette première vague d'immigration. L'auteur rapporte qu'une de ses ancêtres quitta la région de Murcie lorsque son mari, ouvrier agricole en Algérie, fut victime d'un accident de travail. Devant subvenir aux besoins du foyer, la femme investit les économies familiales dans un commerce de vente de charbon à Oran qui ne tarda pas à prospérer. Mais au bout de quelques mois, le mari, se sentant " déplacé ", mit fin à sa convalescence et obligea la famille à déménager vers la campagne pour « reprendre sa place $»^{14}$.

Ces exemples montrent qu'en dépit de l'absence de profession déclarée, les femmes exercent souvent une activité extérieure, ponctuelle ou régulière, à laquelle elles doivent parfois renoncer sous la pression sociale. Le taux de fécondité des Espagnoles (en 1884, le nombre d'enfants était en moyenne de 6,8 dans les couples espagnols, contre 4,4 dans les couples français ${ }^{15}$ ) révèle d'ailleurs la prégnance du modèle patriarcal méditerranéen au sein de la communauté ibérique.

17 Le gouvernement de la II République (1931-1936), premier à s'intéresser au sort de ses ressortissants à l'étranger, commande en 1932 une étude sur la communauté immigrée en Algérie. Les informations apportées par le consulat général d'Alger permettent de mieux appréhender ses conditions d'existence depuis le début du siècle ${ }^{16}$.

18 Dans le département d'Oran, où vivent 60000 Espagnols auxquels s'ajoutent plusieurs milliers de naturalisés (65\% de la population européenne de la région oranaise serait d'origine hispanique), la communauté est omniprésente et travaille dans tous les secteurs de l'économie ${ }^{17}$. Dans le département d'Alger, en revanche, son nombre est 
estimé à trente mille personnes. Originaires des Baléares et du Levant, les Espagnols se consacrent à l'agriculture et à l'horticulture à la campagne et à des emplois de services dans la capitale. Selon le consul, les professions de boulanger, maçon, garçon de café, cordonnier, ébéniste, épicier ou domestique seraient «quasiment monopolisées » par ses compatriotes. Le district d'Orléansville emploie des hommes des provinces de Teruel et de Cuenca travaillant à l'extraction de résine. Les Catalans, enfin, s'installent préférablement dans le département de Constantine, où ils se consacrent à l'exploitation du chêne vert et à des travaux forestiers ${ }^{18}$.

19 Le diplomate souligne que deux types d'émigration co-existent depuis toujours en Algérie: l'une, définitive; et l'autre, temporaire, également appelée golondrina (« hirondelle »).

Los primeros organizan su vida argelina en un medio favorable, ya que los familiares, los paisanos, los amigos acogedores y espontáneos están por regla general prontos a conducirlos y orientarlos. De estos se repatrian pocos, y se fijan en las ciudades, dedicados a menesteres mecánicos o a simples braceros. Solo por razones de salud, o reveses inesperados, vemos a algunos de ellos regresar vencidos a la patria de origen, [...]. En cambio, los segundos que por lo general vienen a épocas determinadas y en grupos para realizar la poda de la viña, naranjos y otros árboles frutales, regresan siempre a sus pueblos de España con el producto de su temporada, siendo raros los que se quedan en el país, como no sea en calidad de encargados de propiedades agrícolas ${ }^{19}$.

20 Le rapport du consul corrobore les conclusions de plusieurs spécialistes : les Espagnols migrent vers l'Algérie via des réseaux régionaux structurés, en raison de facteurs tels que la pauvreté, le besoin d'une main d'œuvre bon marché de la France et la volonté de constituer une colonie de peuplement en Afrique du Nord. La Loi du 26 juin 1889, qui accorde la nationalité française aux enfants nés de parents étrangers sur le territoire national, contribue à enraciner la population hispanique en Algérie. Cependant, une part non négligeable de ses membres continue à privilégier un modèle de migration pendulaire, qui consiste paradoxalement à migrer de façon temporaire pour garder un ancrage dans le pays d'origine, soit à partir pour ne pas partir. Ces deux éléments laissent supposer le maintien de liens forts avec la mère patrie, tout comme de stratégies d'intégration différentes selon le statut de l'immigré.

\section{Années 30-40 : la fin d'un Eldorado}

21 La crise financière qui s'abat sur le monde dans les années 1930 conduit de nombreux pays à fermer leurs frontières et adopter des mesures de protectionnisme social. L'Algérie n'échappe pas à cette tendance. Alors que, jusqu'à cette date, la colonie accueillait la main d'œuvre étrangère sans difficulté majeure, le Décret du 15 mai 1934 et l'Arrêté du 17 avril 1937 qui imposent respectivement la préférence nationale à l'embauche et des quotas de $10 \%$ d'étrangers dans les entreprises privées entraînent un chômage massif qui contraint les Espagnols à retourner au pays ou à entrer dans une situation d'illégalité après plusieurs années de résidence.

22 L'historienne M. E. Cózar Valero remarque toutefois que les femmes sont moins affectées que les hommes par la crise :

Hasta el advenimiento de la II República, se había dejado salir del país a todo aquel que lo solicitaba, sin tener en cuenta la capacidad de absorción del mercado laboral argelino, por lo que todos los años volvían muchos de nuestros emigrantes 
fracasados; no sólo no habían conseguido ahorrar, sino que tampoco habían conseguido un puesto de trabajo. Sólo se permitió salir a aquellos hombres que tuvieran un contrato o bien, en el caso de las mujeres que iban destinadas al servicio doméstico, que hubieran adquirido un billete de ida y vuelta. Estas medidas [...] no se llevaron a la práctica al completo, sobre todo en el caso de la mujer emigrante, pues el éxito de estas en Argelia fue tan grande que pocas regresaron ${ }^{20}$. à de maintes reprises :

Se somete a los españoles, refugiados o residentes legales, a todo género de presiones y coacciones para que firmen formularios bien de enrolamiento o que solamente debieran ser suscritos por asilados y no por ellos que tienen su permiso de residencia. Cuando, aconsejados por el Consulado, se niegan a firmar, se les amenaza con la expulsión en un plazo perentorio [...]. A padres españoles cuyos hijos son franceses, por ser ya la segunda generación nacida en Argelia, se les niega el permiso de trabajo, poniéndoles en el trance de, si quieren repatriarse, tienen que separarse de ellos, pues a estos, ni aun siendo menores de edad, no les expiden pasaportes $^{21}$.

La correspondance consulaire des années 30-40 rend compte de drames humains vécus par les familles espagnoles. Plusieurs missives, écrites généralement par des femmes, implorent ainsi l'aide du consul pour obtenir des nouvelles d'un mari combattant sous les drapeaux français ou interné dans un camp de travailleurs étrangers, le rapatriement d'un fils parti effectuer son service militaire en Espagne et privé de visa de retour par les autorités françaises, ou encore une aide financière car le conjoint parti en Algérie ne subsiste plus aux besoins de sa famille restée en Espagne.

L'afflux de Français fuyant les bombardements en métropole entraîne aussi des réquisitions de logement occupés par des Espagnols. Dans un courrier de 1942 adressé au ministère des Affaires étrangères, Bernabé Toca se plaint de comportements xénophobes à l'encontre de ses compatriotes et cite en exemple le témoignage d'une veuve espagnole au ministère des Affaires étrangères :

Resido en Orán desde el año 1906, tengo 38 años, soy viuda con una hija de 14 años. Mi esposo, español también, era mecánico en la compañía de tranvía de Orán [...]. Vivo en un piso desde 1938, después de la muerte de mi esposo. La dueña de la casa, una señora francesa algo antiespañola, me ha mandado el mes pasado el 'congé' con el fin de que para el día uno de enero le evacuara la habitación. Me dijeron que mi piso era para unos refugiados de Francia que se alojaban actualmente en un hotel. Yo no encuentro casa; inútil será decirle Sr Cónsul lo difícil que es en este momento. [Note du cónsul] La viuda pide un plazo: luego a la calle, o sea a una cueva ${ }^{22}$.

Les travailleurs étrangers ayant conservé leur nationalité d'origine se retrouvent dans une situation d'autant plus précaire qu'ils ne peuvent prétendre aux aides sociales françaises. Privés de travail et de vivres, de nombreux Espagnols se tournent alors vers les œuvres caritatives franquistes, comme l'association de bienfaisance Auxilio Social et 
la Section féminine de la Phalange, particulièrement actives en Oranie dans les années 40-42.

Dans un rapport de ses cinq premiers mois d'activité dans la ville de Sidi-Bel-Abbes ${ }^{23}$, l'Auxilio Social déclare ainsi avoir servi 8000 repas et assuré 42 consultations médicales gratuites. Une permanence juridique et un projet de construction de maternité sont également à l'étude pour venir en aide aux plus démunis. Le même rapport précise qu'au premier semestre 42 , un total de 50000 repas a été distribué par les affiliées des centres d'Oran, Mostaganem, Aïn Temouchent et Perregaux (voir figure 1).

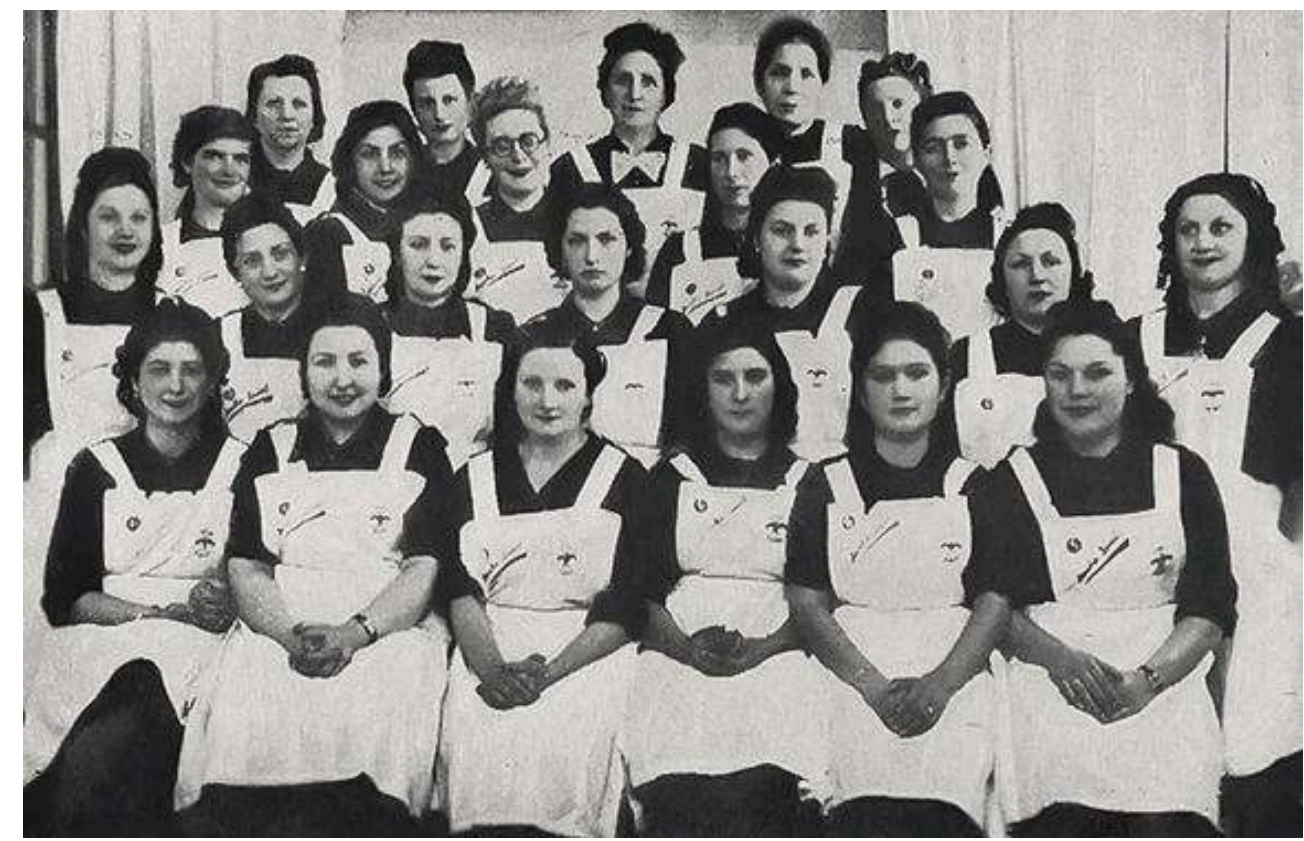

Figure 1. Groupe d'affiliées en charge des services de l'Auxilio Social, Sidi-Bel-Abbes, 1941. Anonyme. AGA, Caja 82/6339. Leg. 2157.

Au regard des pénuries endurées en Espagne, l'aide apportée aux émigrés d'Oranie peut surprendre. Elle répond, en réalité, à des desseins politiques très précis. Outre dénoncer les défaillances du système social français, les autorités franquistes entendent renforcer le lien avec la mère patrie en vue d'entreprendre une nouvelle offensive coloniale en Afrique du Nord :

En la jurisdicción de este consulado, donde reside una minoría española que sobrepasa las 20.000 almas, no podía estar ausente esta gran obra nacional que encarna el espíritu renovador y revolucionario de la Nueva España y que ha de llevar a estos compatriotas, antes tan separados moral y materialmente de la Patria, su aliento, su calor y su fe absoluta y firme en su renacer Imperial ${ }^{24}$.

Considérant que l'Oranie est, depuis la conquête de Cisneros en 1509, un territoire « hispanique », Franco entend profiter de l'entrée en guerre de la France pour annexer ce territoire ${ }^{25}$. L'Auxilio Social et la Section Féminine relayent donc une propagande irrédentiste, qui dénonce le traitement réservé à la main d'œuvre étrangère, établit une distinction entre «bons Espagnols» et "renégats» (c'est en ces termes que les naturalisés antifranquistes sont désignés ${ }^{26}$ ) et incite les femmes à aller accoucher en Espagne pour éviter la naturalisation automatique de leurs enfants. Conséquence ou non de cette propagande, il n'est pas rare de voir au sein d'une même fratrie que des enfants naissent de part et d'autre de la Méditerranée. Les récits mémoriels insistent, 
d'ailleurs, sur le fait que les femmes (gardiennes de la langue et des traditions espagnoles) entretiennent volontairement les liens affectifs avec la péninsule.

31 La période retenue pour cette étude correspond donc à la fin de l'El Dorado algérien pour les immigrés espagnols. Victimes la récession économique des années 30 et d'une législation discriminante pendant la guerre, beaucoup sont contraints de regagner leur pays ou de changer de secteur d'activité afin de contourner les quotas à l'embauche. Le retour de la paix ne s'accompagne pas d'une nouvelle vague d'immigration. Au contraire, suite à l'insurrection nationaliste algérienne de 1945, les autorités françaises encouragent le remplacement des travailleurs non-français par des Musulmans dans différents secteurs ${ }^{27}$. Rosa Montero explique, à ce propos, que les domestiques musulmanes firent leur entrée dans les foyers européens au moment de l'Après-guerre, et le Consulat d'Espagne que «les tailleurs de vigne d'Alicante, qui venaient périodiquement en Oranie, furent progressivement remplacés par la main d'œuvre indigène ». Les migrations économiques se tarissent donc au profit des migrations politiques.

\section{L'exil politique}

La fin de la Guerre civile espagnole suscite d'importants mouvements de population en direction de l'Algérie à partir de février 1939. Embarqués sur des bateaux dont le nom est passé à la postérité (le Stanbrook, le Ronwyn, l'African Trader, le Lezardieux), dix mille républicains sont évacués des ports d'Alicante et de Valence en quelques semaines. Craignant que l'arrivée de ces réfugiés ne déstabilise l'ordre social précaire de la colonie, les autorités françaises décident de les refouler vers la France ou l'Amérique du $\mathrm{Sud}^{28}$. Mais leur nombre est tel qu'ils sont finalement autorisés à rester et placés en centres de rétention ou chez l'habitant.

Un recensement effectué par le gouverneur général d'Oran en juin 1939 apporte des précisions quant au nombre et au profil des réfugiés. Sur 2750 individus recensés dans ce département, 1706 sont internés dans des camps civils ou militaires et 1050 hébergés chez des particuliers ayant un lien de parenté avec ces derniers. Constituée à $67 \%$ d'hommes, $25 \%$ de femmes et $8 \%$ d'enfants, cette population est souvent issue du prolétariat urbain des provinces de Valence, Alicante, Murcie et Carthagène ${ }^{29}$ et présente des caractéristiques sociologiques différentes de l'émigration économique: davantage masculine, politisée et urbaine, elle s'établit préférablement dans les villes d'Oran et d'Alger.

Peu d'informations sont disponibles sur les migrantes. La liste nominative des passagers du Stanbrook, conservée aux Archives nationales d'Outre-mer d'Aix-enProvence, permet d'élaborer une première ébauche de portrait. Les 300 femmes adultes embarquées sur ce bateau ont en moyenne 29 ans et voyagent plutôt en famille. Presque la moitié déclare avoir une profession: modistes (12\%), dactylos (8\%), journalières ou tailleuses (4\%), institutrices (3\%), infirmières (2\%), étudiantes (2\%), sandalières $(2 \%)^{30}$. Les républicaines présentent donc un niveau d'instruction légèrement supérieur à celui des immigrées économiques. Néanmoins, la poursuite de leur activité professionnelle se trouve compromise durant les premières années d'exil.

Regroupées avec leurs enfants dans des centres de rétention comme l'ancienne prison civile d'Oran, les républicaines vivent dans des conditions sanitaires déplorables qui 
font craindre au préfet un "risque de déficience vitale ${ }^{31}$ ». Après la fermeture de ce centre à l'hiver 1940, elles sont évacuées vers d'autres lieux d'hébergement où le regroupement familial est autorisé sauf en cas de «dangerosité sociale » du conjoint. Les conditions de vie en Algérie diffèrent donc en fonction de l'appartenance politique des chefs de famille : tandis que socialistes et élus républicains bénéficient d'un retour plus rapide à la vie civile grâce, notamment, à la présence de leur famille sur le territoire, communistes et anarchistes sont séparés de leurs proches et internés dans des camps où prévalent des conditions inhumaines, tel que le relatent l'écrivain Max Aub dans son recueil de poèmes Diario de Djelfa et l'anarchiste José Congost Muñoz, dans le récit mémoriel Por tierras de moros, sur son internement à Hadjerat M'Guil ${ }^{32}$.

En 1943, les réfugiés encore retenus dans des camps sont libérés. Certains choisissent de poursuivre leur exil au Mexique ou en Union Soviétique. D’autres décident de rester en Algérie, où ils peuvent compter sur la solidarité de leurs compatriotes et anciens partis. Selon un recensement effectué par le consulat, quatre organisations sont reconstituées à Oran pendant la Seconde guerre mondiale: Union générale des travailleurs-Parti socialiste ouvrier espagnol (7.000 affiliés), Parti communiste espagnol (4.000), Confédération nationale du travail-Fédération anarchiste ibérique (2.400) et le parti de centre-gauche de l'ancien président Azaña, Izquierda Republicana (400) ${ }^{33}$. Le PCE est, de loin, le plus structuré. Il bénéficie du soutien des partis communistes soviétique, français et algérien, comme l'atteste la photographie de la figure 2, prise au cours d'une manifestation de soutien au Front Populaire espagnol.

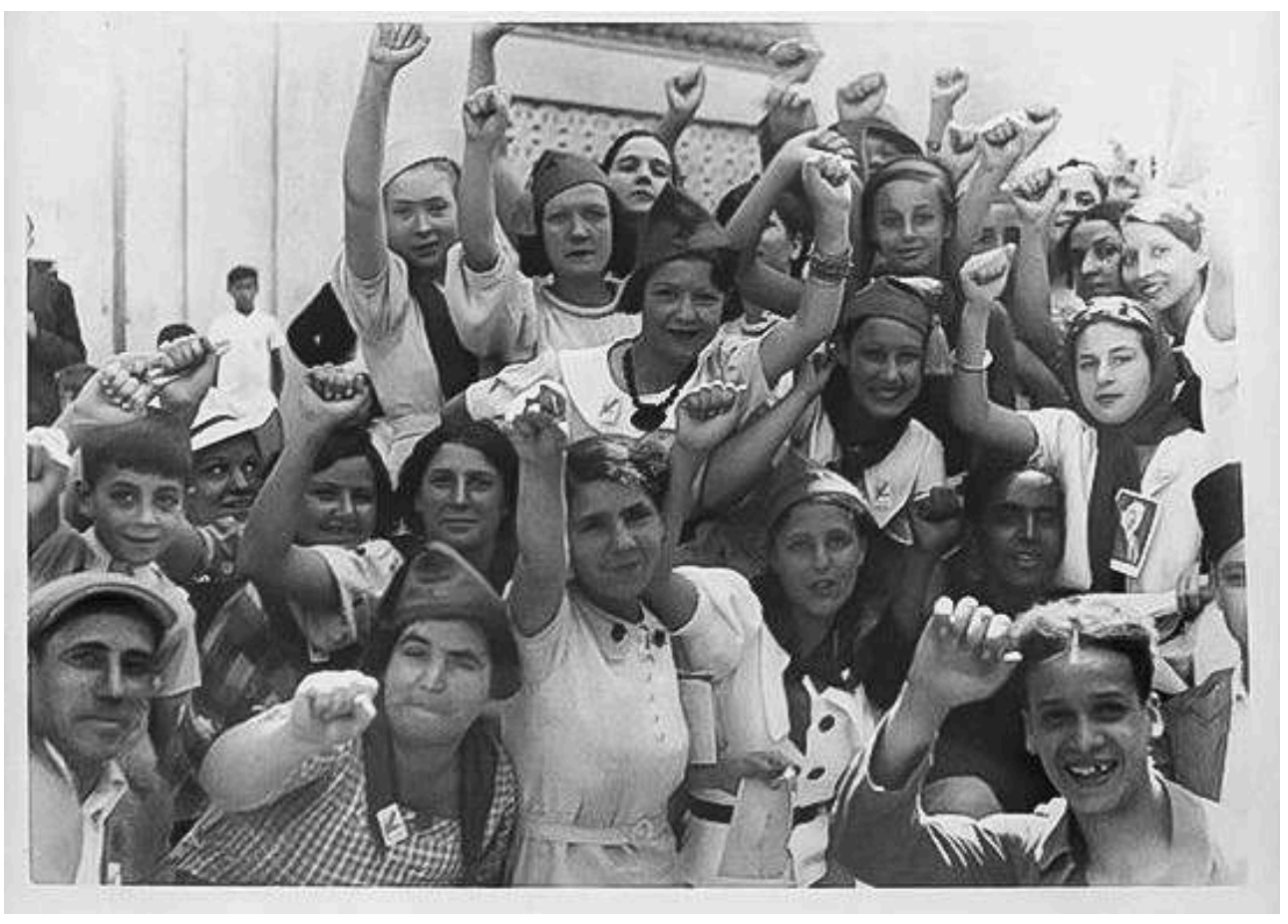

Figure 2. 14 juillet 1937, Alger. Les jeunesses communistes manifestent en faveur du Front populaire espagnol. Anonyme. Biblioteca Nacional de España.

37 Le syndicaliste Marcelino Camacho, évadé de la prison franquiste de Tanger, relate dans ses mémoires l'ambiance qui règne à Oran à la fin des années 40 . Après avoir été hébergé chez un ancien brigadiste international et un réfugié, il rencontre Josefina, couturière espagnole dont la famille a immigré d'Almería en 1931, avec qui il se marie en 1947. Vivant à l'instar de beaucoup d'Espagnols dans un logement modeste (une 
cuisine et une chambre pour l'ensemble de la famille, des toilettes et des douches dans les parties communes) du quartier de Gambetta, le quotidien du couple est marqué par le travail à l'usine ou à l'atelier et les fêtes familiales et les réunions politiques entre réfugiés le week-end :

Josefina y yo salimos muchos domingos con otros camaradas a vender la prensa y propaganda del partido [...]. Otras veces vendíamos flores y hacíamos una especie de cuestación con huchas, para recoger dinero al tiempo que se organizaba algún mitin o asamblea, especialmente en días como el Primero de Mayo u otras celebraciones. En muchas ocasiones, hacíamos esas reuniones en protesta por la represión en España o por las condenas a muerte que periódicamente se sucedían por aquellos años ${ }^{34}$.

Dans les rapports de surveillance des autorités consulaires, l'activité des républicaines n'est jamais évoquée. On constate pourtant qu'entre 1947 et 1949, un grand nombre de femmes sollicitent un passeport ou un visa pour voyager de l'Espagne vers l'Algérie ou inversement. $72 \%$ des demandes rejetées concernent d'ailleurs des femmes ${ }^{35}$. On peut donc supposer que l'ouverture de la frontière franco-espagnole et les amnisties politiques de 1947 incitent certaines d'entre elles à rejoindre leur mari exilé en Algérie ou retourner en Espagne, où leurs enfants sont restés lors de la débâcle républicaine.

La Circulaire franquiste 2454 du 23 novembre 1954, qui autorise le retour définitif ou temporaire des réfugiés n'ayant pas de condamnation pénale en cours en Espagne, entraîne de nouvelles demandes de visas. Arguant pour la plupart qu'elles ont « suivi leur mari » en 1939 et qu'elles souhaitent « rendre visite à leurs proches » (comme nous pouvons l'apprécier dans la figure 3), les femmes sont les premières à bénéficier de ces mesures de clémence qui se consolident en 1956. Dans une note adressée à l'ambassade d'Alger, la Sûreté de l'État affirme, en effet, que « sauf en cas de dangerosité politique notoire de leur mari, le fait d'être épouse d'un exilé politique ne sera pas motif d'entrave à l'obtention de visa ${ }^{36} »$. 

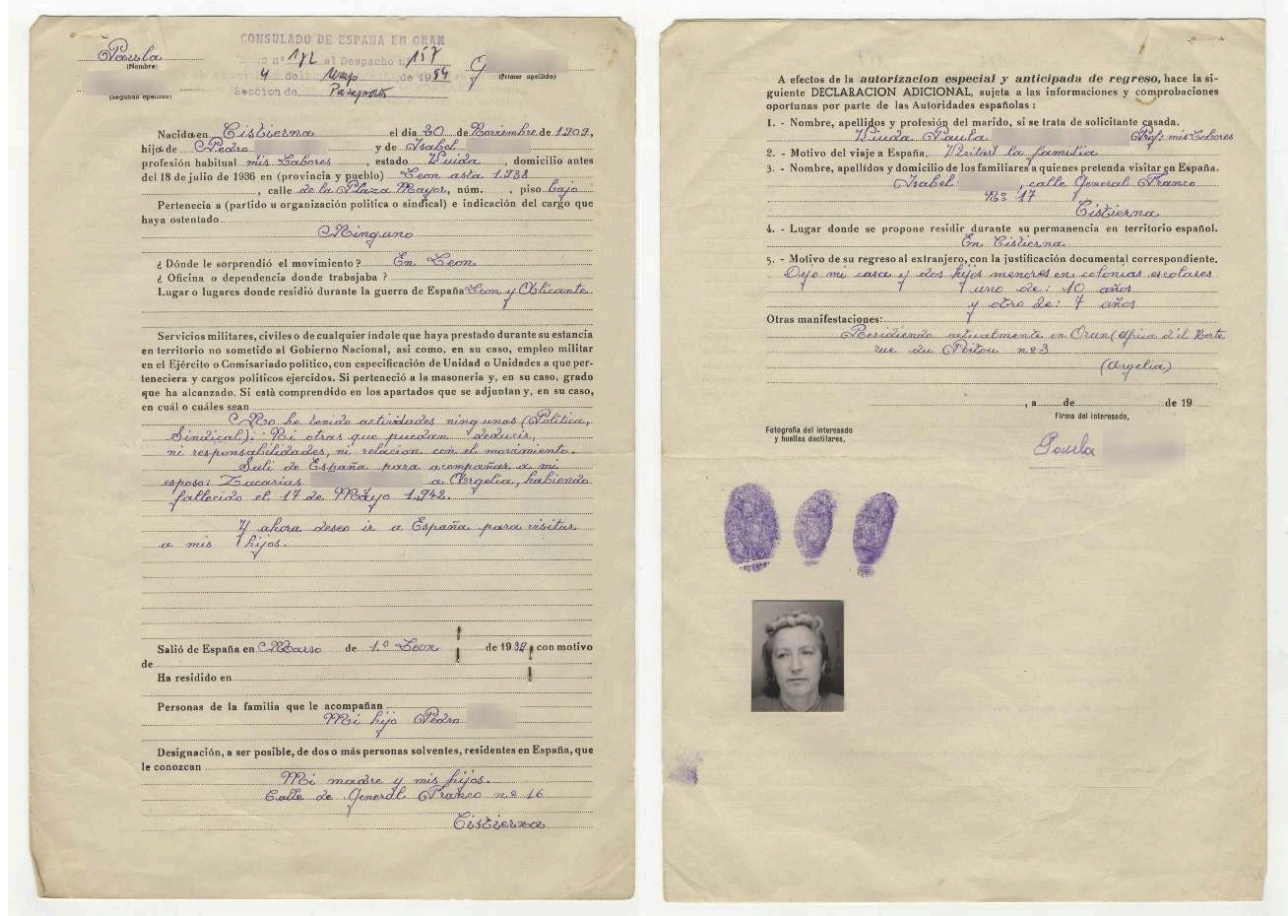

Figure 3. Formulaire d'autorisation de retour en Espagne, adressé par la réfugiée Paula G. aux autorités consulaires d'Oran, 4 mai 1954. Source : AGA.

Ignorant le fait que les femmes puissent être animées d'une conscience politique (nous pourrions objecter que la réfugiée Carmen Consuegra Linares avait recréé une section du Parti socialiste ouvrier espagnol dans la ville de Colomb Béchard et participé au III $^{\mathrm{e}}$ Congrès des Jeunesses Socialistes à Oran en $1947^{37}$ ), le pouvoir franquiste semble donc moins vigilant sur le retour des femmes, hormis lorsque celles-ci sont mariées à des communistes. Pour autant, rares sont les réfugiés à solliciter un retour définitif : sur 150 demandes déposées auprès du consulat d'Oran entre 1954 et 1962, seuls $10 \%$ évoquent cette possibilité. Et cela, malgré les consignes du PCE de regagner la péninsule pour y préparer la « lutte intérieure $»^{38}$.

41 Le déclenchement de la guerre d'indépendance ne semble pas, non plus, être un élément déclencheur de retour jusqu'en 1961. À l'instar des autres membres de la communauté européenne, les Espagnols choisissent majoritairement de rester en Algérie jusqu'aux dernières heures de la colonie et d'être ensuite rapatriés vers la France. De nombreux courriers adressés au consulat indiquent, par exemple, qu'ils ne souhaitent pas être séparés de leurs enfants qui ont acquis la nationalité française. Contrairement aux étrangers résidant dans la colonie, ces derniers peuvent d'ailleurs escompter une meilleure indemnisation des biens nationalisés en Algérie (indemnisation dont sont privés les Espagnols ayant conservé leur nationalité).

Le chiffre de 30000 rapatriés accueillis en Espagne en 1962 semble donc exagéré, même s'il est vrai que les autorités franquistes contribuent à l'évacuation de ressortissants espagnols et français en juillet 1962. La veille du massacre d'Oran, 2111 passagers sont ainsi embarqués vers Alicante, à bord du Virgen de África et du Victoria ${ }^{39}$. Les retours vers la mère patrie sont donc finalement assez peu nombreux au regard du nombre total d'Européens vivant en Algérie. 


\section{Conclusion}

43 À rebours des clichés sur la victimisation des femmes migrantes, cette première approche de l'émigration des Espagnoles en Algérie montre que celles-ci prirent une part active au phénomène migratoire, faisant preuve de la même détermination et résistance que leurs compatriotes masculins. Sortant du foyer et de leur communauté d'origine pour répondre aux besoins de main d'œuvre de la société d'accueil ou pour seconder leur conjoint dans l'aventure coloniale, les migrantes contribuèrent non seulement à l'essor économique et démographique de l'Algérie mais aussi à son identité culturelle. Si les rapports hiérarchiques, ethniques et sexués de l'Algérie des années 30-60 n'entraînent pas nécessairement une redéfinition du rôle social qui leur était assigné en Espagne, des opportunités d'émancipation financières existent, notamment pour les résidentes permanentes - moins sujettes aux fluctuations économiques que les saisonnières. Néanmoins, il est encore totalement prématuré de parler de prise de pouvoir des femmes.

La vision androcentriste du réel a pour conséquence une relative absence des femmes dans les archives de l'administration. Celles-ci apparaissent lorsque l'intervention des autorités consulaires s'avère nécessaire pour secourir un proche, obtenir une aide matérielle ou faciliter un regroupement familial. En revanche, leur condition de citoyenne politique n'est presque jamais évoquée, comme si l'obtention du droit de vote et l'investissement de l'espace public par les femmes durant la $\mathrm{II}^{\mathrm{e}}$ République n'avaient jamais existé. La consultation d'archives politiques et syndicales permettrait sans doute de démontrer que les exilées n'étaient pas que de simples "suiveuses ».

C'est en tout cas ce qu'affirme l'écrivain Manuel Vázquez Montalbán à propos des épouses et compagnes de républicains. Ces femmes, dit-il, avaient souvent une double militance: "celle qui les amenait à s'engager dans la grande cause générale et universelle de l'émancipation humaine; et celle, de premier plan, sentimentale, qui les reliait par un cordon ombilical invisible à l'homme qu'elles aimaient $»^{40}$.

\section{NOTES}

1. Albert Camus, "Actuelles III ", in Chroniques algériennes, p. 207.

2. Selon le géographe Kamel Kateb, le recensement de 1954 fait état de 984031 Européens et de 8 449332 « indigènes » ou musulmans.

3. Flores, Christian. Un siècle de colonisation hispanique en Oranie française (1830-1930), Mémoire de maîtrise, Université d'Angers, 1980. Jordi, Jean-Jacques. Les Espagnols en Oranie : 1830-1914 : histoire d'une migration, Montpellier, Africa Nostra, 1986. Crespo, Gérard et Jordi, Jean-Jacques. L'immigration espagnole dans l'Algérois : de 1830 à 1914, Versailles, Atlantrophe, 1990. Dulphy, Anne. L'Algérie des Pieds-noirs : entre l'Espagne et la France, Paris, Vendémiaire, 2013.

4. Citons notamment Vilar, Juan Bautista. Los españoles en la Argelia francesa (1830-1914), CSIC, Universidad de Murcia, 1989. 
5. Menages Ángela-Rosa y Monjo, Joan- Lluis. Els valencians d'Algeria (1830-1962), Picanya, Edicions del Bullent, 2007. Buades Crespí, Joan. L'emigració balear a ultramar (1830-1960), Palma, Documenta Balear, 2009. Olives Galmés, Llorenç. La migració menorquina a Algèria : visions d'un trescador entre Menorca i El Magreb, Alaior, Olves Galmés, 2011. Gilabert Marqués, Gabriel y Pascual Bolufer, Antoni. Anar a Orà: l'emigració del Poble Nou de Benitatxell a l'Algèria francesa, Picanya, Edicions del Bullent, 2009. Marfany, Marta. L'emigració menorquina a Algèria al segle XIX, Palma, Documenta Balear, 2015

6. Catarino, Christine et Morokvasic, Mirjana. « Femmes, genre, migration et mobilité », Revue européenne des migrations internationales, vol. 21-1, 2005, p. 7-27.

7. Robert-Guiard, Claudine. Des Européennes en situation coloniale, Aix-en-Provence, Université de Provence, 2009 [version E-book]. L'italique est de nous.

8. Robert-Guiard, Claudine. Des Europénnes..., op. cit., p. 15.

9. Verdès-Leroux. Jeannine. Les Français d'Algérie: De 1830 à aujourd'hui. Une page d'histoire déchirée, Paris, Fayard, 2001, p. 206.

10. Bugeaud à Genty de Bussy, Alger, le 30 mars 1847, Lettres inédites, p. 304. Ibidem, p. 190.

11. Montero. Andrée. Río Salado, Toulouse, Privat, 1981.

12. Victor Demontès, p. 62-65, note qu'il y avait en 189693 hommes pour 100 femmes chez les Espagnols; 120 hommes pour 100 femmes chez les Français, 116 hommes pour 100 femmes dans l'ensemble de la communauté européenne. Verdes-Leroux, Jeannine. Les Français d'Algérie..., op. cit. p. 207.

13. De la prostitution dans la ville d'Alger depuis la conquête, Paris, 1953, p. 58.

14. Núñez, Denis. Les golondrinas, ou les trois sceurs d'Almería, Paris, L'Harmattan, 2013, p. 226.

15. Robert-Guiard, Claudine. Des Européennes..., op. cit., p. 140.

16. AGA, MAE. Caja 82/2847. Leg R721. Exp. 41. «Intereses demográficos 1932 ».

17. Nous ne disposons pas des chiffres de 1931 mais ils étaient, d'après le recensement de 1936, 66000 en Oranie. A. Dulphy, L'Algérie des pieds-noirs, op. cit., p. 21.

18. AGA, MAE. Caja 82/2847. Leg R721. Exp. 41. «Intereses demográficos 1932 ».

19. Ibidem.

20. Cózar Valero, María Enriqueta. La emigración exterior de Almería, Granada, Universidad, 1984, p. 83.

21. AGA, MAE, Caja 66/4167. Correspondencia 66. 14/10/39.

22. Ibidem. Correspondencia 7. 11/2/1942.

23. Caja 82/6339. Leg. 2157. Exp.1 « Labor de la Falange en Argelia ».

24. Ibidem.

25. Salinas, Alfred. Quand Franco réclamait Oran. L'opération Cisneros, Paris, L'Harmattan, 2008.

26. Dulphy, Anne. L'Algérie des Pieds-Noirs, op. cit., p. 169.

27. Traduction de l'auteure. AGA, MAE, Caja 4157.

28. Selon les décrets Daladier, les étrangers "indésirables" doivent être expulsés, rapatriés ou internés.

29. A. Dulphy, L'Algérie des Pieds-Noirs..., op. cit., p. 87.

30. Vilar, Juan Bautista. « Relación nominal de los militantes republicanos evacuados de Alicante por el buque inglés Stanbrook con destino a Oran en 28 de marzo de 1939 ", Anales de historia contemporánea, n²2, Universidad de Murcia, 1983, p. 273-330. Sont considérées comme adultes les femmes de plus de 30 ans.

31. A. Dulphy, L'Algérie des Pieds-Noirs..., op. cit., p. 90.

32. Aub, Max, Diario de Djelfa (1941-1942). México, Joaquín Mortiz, 1970. Muñoz Congost, José. Por tierras de moros: el exilio español en el Magreb, Madre Tierra, Móstoles, 1989. 
33. Informe sobre la política de los españoles refugiados en Orán. 20/12/40. AGA 82-6487. MAE. Leg 2222. Exp. 51. 1942/1944.

34. Camacho, Marcelino. Confieso que he luchado, Madrid, Temas de Hoy, 1990 p. 120.

35. Pourcentages établis à partir d'une liste nominative de passeports et visas refusés. AGA, MAE, Caja 66/4157. Un tiers des demandes concernent des autorisations de voyage vers l'Algérie.

36. AGA. MAE. Caja 66/51 47. Orden 138 de 30 de agosto 1956. Traduction de l'auteure.

37. Source: Fondation Pablo Iglesias.

38. Camacho, Marcelino. Confieso..., op. cit., p. 134.

39. AGA, MAE, Caja 66/4138.

40. Camacho, Marcelino. Confieso..., op. cit., p. 17. Prologue. Traduction de l'auteure.

\section{ABSTRACTS}

Cet article porte sur l'immigration féminine espagnole en Algérie entre 1939 et 1962. À partir de sources issues des Archives Générales de l'Administration espagnole (AGA) d'Alcalá de Henares et de différents récits mémoriels, il tente d'identifier les facteurs ayant entraîné le départ vers l'Algérie, ainsi que les conditions d'intégration de ces femmes au sein de la société d'accueil. Après avoir rappelé les grandes caractéristiques de l'immigration espagnole depuis la conquête militaire de l'Algérie, nous verrons que ce territoire cesse, dans les années 1930, de constituer un Eldorado pour les migrants; et que l'intégration des Espagnoles diffère selon leur condition d'immigrées économiques ou d'exilées politiques républicaines. Cette contribution constitue une première approche sur les migrations féminines espagnoles en Afrique du Nord.

\section{INDEX}

Chronological index: XXe siècle

Subjects: Migrations, femmes, Espagne

Mots-clés: Migrations, genre, exil républicain, Algérie, Espagne

Geographical index: Espagne, France, Algérie

\section{AUTHOR}

\section{LUDIVINE THOUVEREZ}

Université de Poitiers, MimmocDocteure en Etudes Ibériques et en Sciences de l'information et de la communication, Ludivine Thouverez est Maîtresse de Conférences en Espagnol à l'Université de Poitiers. Ses recherches portent sur l'Espagne contemporaine et la construction médiatiques des conflits. 